\section{Cyst infection in acquired renal cystic disease}

Cystic degeneration of the kidney in patients with advanced renal failure is common'; whether cysts can harbour infection remains to be shown. ${ }^{2}$ We report on three cases of infected cysts in acquired renal cystic disease.

\section{Case reports}

Case 1-A 60 year old man was admitted to hospital because of uraemia, hypertension, recurrent fever, and leucocytosis. The plasma concentration of creatinine was $821 \mu \mathrm{mol} / \mathrm{l}$, and a urine culture was positive for Escherichia coli. Ultrasonography showed normal sized hyperechogenic kidneys with more than three $1-2 \mathrm{~cm}$ cysts in each. There was a $6 \mathrm{~cm}$ cyst that was hyperechogenic at the upper pole of the left kidney. Renal computed tomography confirmed the presence of bilateral multiple cysts. A thick wall, enhanced by contrast infusion, was seen around a slightly dense cyst on the upper pole of the left kidney (figure).

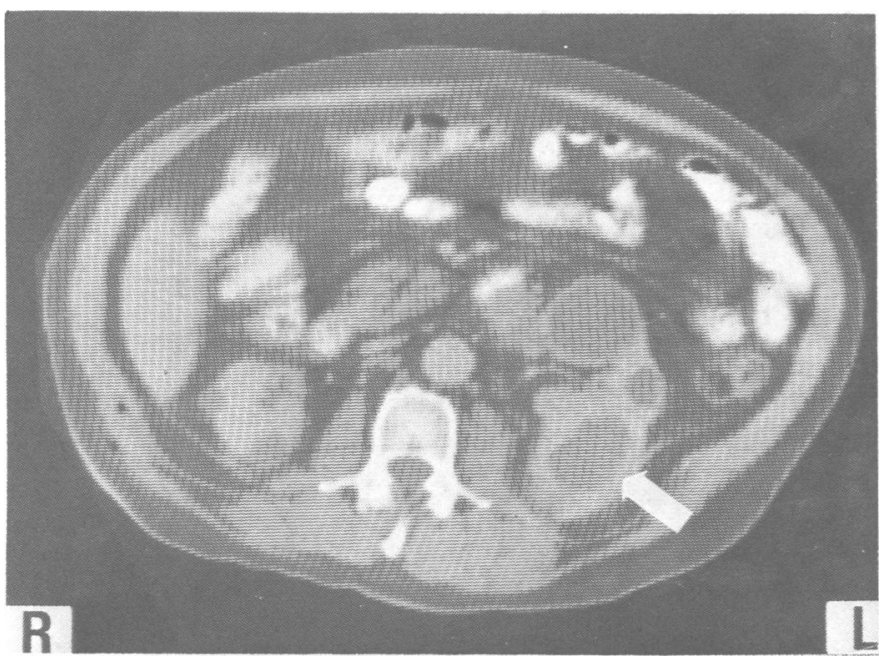

Both kidneys are occupied by low density cysts. A thick wall, enhanced by contrast infusion, is seen around a slightly dense cyst (arrow).

Renal biopsy showed chronic glomerulonephritis with mesangial deposits of C3. Percutaneous aspiration of the cyst yielded purulent fluid which was positive for $E$ coli. He was given tobramycin; the fever disappeared, and the urine became sterile and the white cell count normal.

Case 2-A 60 year old woman with renal talure secondary to diabetic nephropathy was admitted to hospital because of fever and dysuria. Plasma creatinine concentration was $482 \mu \mathrm{mol} / \mathrm{l}$, she had leucocytosis and pyuria, and a urine culture was positive for $E$ coli. A radiograph of the abdomen showed air in the left renal profile. Ultrasonography showed multiple renal cysts and a hyperechogenic parenchyma. Computed tomography confirmed the presence of the cysts and suggested an abscess at the lower renal pole and perirenal space of the left kidney. We removed a cyst, the fluid of which was infected by $E$ coli. Tobramycin was administered and the white cell count became normal and the urine sterile.

Case 3-A 78 year old woman presented with uraemia and creatinine values of $1120 \mu \mathrm{mol} / \mathrm{l}$ and was started on haemodialysis. Ultrasonography showed small, hyperechogenic kidneys with multiple cysts. On admission she had a fever with no apparent cause. No urine cultures were available. She died a few days later of haemorrhage of the upper digestive tract. Necropsy showed two end stage kidneys with multiple corticomedullary cysts; an infected cortical cyst was found in the left kidney.

\section{Comment}

Although acquired renal cystic disease was initially described in patients undergoing haemodialysis, it can also affect patients with long term advanced renal failure. ${ }^{3}$ It is generally without symptoms but may present with pain associated with haemorrhage or neoplasia, or both. Infected cysts have not been reported before in acquired renal cystic disease. ${ }^{4}$

Cyst infection is a rare complication of simple renal cysts, and the incidence of cyst infection in adult polycystic kidney disease has not been established. In either case patients have pyuria and most have a history of urinary tract infection. The infection is usually persistent and often progresses despite adequate antibiotic treatment because the antibiotic reaches the cystic fluid in only small amounts.

Differentiation of an infected cyst from a non-infected one may be difficult by ultrasonography: renal ultrasonography shows the presence of internal echos, and computed tomography detects thick walls or slightly dense cysts. A diagnosis can be confirmed only by puncturing the cyst. Continuous antibiotic treatment with percutaneous drainage of the cyst guided by ultrasonography or computed tomography is an alternative to surgical drainage and antibiotic treatment. ${ }^{5}$ Infected cysts should be suspected in patients with acquired cystic disease and persistent fever.

1 Ratcliffe PJ, Dunnill MS, Oliver DO. Clinical importance of acquired cystic disease of the kidneys in patients undergoing dialysis. Br Med $\mathcal{F}$ 1983;287:1855-8.

Gardner KD, Evans AP. Cystic kidneys: an enigma evolves. Am 7 Kidney Dis 1984;3:403-13. 3 Bommer J, Waldherr $R$, Van Kaick G, Strauss L Ritz E. Acquired renal cysts in uremic patients. In vivo demonstration by computed tomography. Clin Nephrol 1980;14:299-303.

4 Narasimban N, Golper TA, Wolfson M, Rahatzad M, Bennet WM. Clinical characteristics and diagnostic considerations in acquired renal cystic disease. Kidney Int 1986;30:748-52.

5 Finn DJ, Palestrant AM, Dewolf WC. Successful percutaneous management of renal abscess. f Urol 1982;127:425-6.

(Accepted 17 March 1987)

Servei de Nefrologia, Hospital de Badalona "Germans Trias i Pujol," Badalona, Spain

JORDI BONAL, MD, nephrologist

ANTONIO CARALPS, MD, nephrologist, head of the service

RICARDO LAUZURICA, MD, nephrologist

ASSUMPTA SERRA, MD, nephrologist

RAMON ROMERO, MD, nephrologist

Servei de Radiologia, Hospital de Badalona

LUIS INARAJA, MD, radiologist

Correspondence to: Dr Bonal.

\section{Neonatal respiratory distress caused by aspiration of a vaginal tablet containing prostaglandin}

The use of prostaglandin $E_{2}$ for cervical ripening and induction of labour is becoming more common. It may be given in a viscous gel, ${ }^{1}$ vaginal tablets, ${ }^{2}$ or pessaries ${ }^{3}$ and is considered both safe and effective. ${ }^{1.4} \mathrm{We}$ report a case of neonatal aspiration syndrome caused by a vaginal tablet containing prostaglandin.

\section{Case report}

A 28 year old woman who had had a caesarean section three years previously was admitted to hospital at $\mathbf{4 0}$ weeks of pregnancy because of onset of labour External cephalic version had been performed without complications at 38 weeks' gestation; otherwise the course of the pregnancy had been uneventful. The cervix was ripe, and the baby's head was $2 \mathrm{~cm}$ above the ischial spines. A fetal cardiotocogram was normal. Because the patient was two days post term and the contractions remained irregular after six hours' observation, a vaginal tablet containing $3 \mathrm{mg}$ of prostaglandin $\mathrm{E}_{2}$ (Prostin E2) was inserted into the posterior vaginal fornix. Uterine contractions improved, and $131 / 2$ hours later the cervix was $6 \mathrm{~cm}$ dilated. After amniotomy a normal amount of clear amniotic fluid escaped. Cardiotocography showed normal reactivity, but occasional, early decreases in fetal heart rate were also seen. Fifteen hours after insertion of the vaginal tablet the cervix was fully dilated, and $\mathbf{4 0}$ minutes later a girl weighing $3190 \mathrm{~g}$ was born in the normal vertex position. The umbilical cord was around the neck and was cut immediately after delivery of the head.

The baby had apnoea and bradycardia and an Apgar score of 5 at one minute. After receiving oxygen by mask her condition improved slightly, and at 10 minutes she had an Apgar score of 7. Her breathing remained irregular, however, and she began to cough; a white granular mass was seen in the mouth and pharynx. Despite suction of the upper airways respiration remained difficult, and the child was intubated at 11 minutes. An additional white mass resembling a tablet was suctioned from the lower airways until extubation $\mathbf{4 0}$ minutes after 